\title{
Editorial \\ Digital Psychology as a Growing Field of Research
}

\author{
Oswald D. Kothgassner ${ }^{1} \&$ Anna Felnhofer ${ }^{2}$ \\ ${ }^{1}$ Department of Child and Adolescent Psychiatry, Medical University of Vienna \\ 2 Department of Pediatrics and Adolescent Medicine, Medical University of Vienna
}

DOI 10.24989/dp.v1i1.1852

The Journal Digital Psychology is conceptualized as a scholarly journal and a platform for knowledge transfer at the interface between digitalization, new media, and psychology as well as related disciplines (e.g. psychiatry, communication science). The key topics of the journal include current research, applied science and practice, and upcoming technological developments in the field.

Digital Media in Psychology is a rapidly growing field and there is a strong need for increased focus on research synthesis to strengthen scientific evidence for emerging theories, methods and therapeutic interventions. Therefore, Digital Psychology aims on the one hand to highlight qualitative and quantitative reviews as well as practitioner reviews (opinions, clinical practice), and on the other hand to foster discussion and exchange about pertinent topics and issues in this developing area of research. Furthermore, we welcome research on the influence of technology on children and adolescents, with a particular focus on clinical, social, and economic impacts. We sincerely hope that these topics will become an integral part of our journal.

However, the journal's scope is not limited to these subjects. We also invite scholars working on other topics in the broader field of Digital Psychology (e.g. e-Learning, computer-based assessments, computer-mediated communication, Virtual Reality/ Augmented Reality) to submit their research.

The journal comprises two issues per year and includes articles (reviews and original research, letters, and spotlight-communications) in English and in German. Professionals with an interest in a psychological perspective on digital media will find this journal to be of great interest.

Digital Psychology will provide rigorous peer-review by experts in the field and an editorial board covering a broad range of research topics. Moreover, this journal will be archived in PubMed Central and other full-text repositories.

We strongly believe that Digital Psychology is a valuable addition to the existing journal landscape on digital media. We are convinced that our collaboration with authors of high quality research, esteemed expert reviewers, and an engaged readership will ensure the success of this journal. We hope that Digital Psychology will provide not only a platform for open discussion, but also create fruitful ground for promoting novel insights and developments in this ever-changing area of research.
We thank our Editorial Board Team, as well as the Production Editors and our publisher for the hard work and support that made this adventurous enterprise possible.

Oswald D. Kothgassner and Anna Felnhofer Editors-in-Chief

Declaration of Interests: The authors declare that they have no competing interests.

\section{Table of Contents}

\section{Editorial}

2 Expert Views on the Future of Digital Media in Psychology

4 Development and Usability Testing of SOMO, a Mobile-Based Application to Monitor Social Functioning for Youth at Clinical High-Risk for Psychosis Olga Santesteban-Echarri, Jacky Tang, Jaydon Fernandes \& Jean Addington

20 Frequency and Duration of Daily Smartphone Usage in Relation to Personality Traits Felix Beierle, Thomas Probst, Mathias Allemand, Johannes Zimmermann, Rüdiger Pryss, Patrick Neff, Winfried Schlee, Stefan Stieger \& Sanja Budimir

29 Digital Family Life: A Systematic Review of the Impact of Parental Smartphone Use on Parent-Child Interactions Barbara Knitter \& Martina Zemp

44 Digital Phenotyping - A Case for Cognitive Functions and Dementia? Christian Montag \& Jon D. Elhai

52 Digital Phenotyping of Big Five Personality Traits via Facebook Data Mining: A Meta-Analysis Davide Marengo \& Christian Montag 